IRA-International Journal of Education \&

Multidisciplinary Studies

QUARTERLY

ISSN 2455-2526; Vol.16, Issue 03 (July-Sep, 2020)

Pg. no. 181-188.

Institute of Research Advances

https://research-advances.org/index.php/IJEMS

IRA

\title{
Vietnamese Students' Perceptions toward the Use of GeoGebra in the Learning of Mathematics
}

\author{
Le Viet Minh Triet $^{1 \#}$, Nguyen Phu Loc ${ }^{2}$ \\ ${ }^{1}$ Department of Mathematics, Ho Chi Minh City University of Education, Vietnam. \\ ${ }^{2}$ School of Education, Can Tho University, Vietnam. \\ \#corresponding author \\ Type of Work: Peer-Reviewed \\ Received: September 03, 2020 Revised: September 14, 2020 Accepted: September 23, 2020 \\ DOI: http://dx.doi.org/10.21013/jems.v16.n3.p7 \\ How to cite this paper: \\ Triet, Le Viet Minh et al. (2020). Vietnamese Students' Perceptions toward the Use of GeoGebra in \\ the Learning of Mathematics. IRA International Journal of Education and Multidisciplinary Studies (ISSN \\ 2455-2526), 16(3), 181-188. DOl: http://dx.doi.org/10.21013/jems.v16.n3.p7
}

(C) Institute of Research Advances.

This work is licensed under a Creative Commons Attribution-NonCommercial 4.0 International License subject to a proper citation to the publication source of the work.

Disclaimer: The scholarly papers as reviewed and published by the Institute of Research Advances (IRA) are the views and opinions of their respective authors and are not the views or opinions of the IRA. The IRA disclaims of any harm or loss caused due to the published content to any party.

Institute of Research Advances is an institutional publisher member of Publishers International Linking Association Inc. (PILA-CrossRef), USA. The institute is an institutional signatory to the Budapest Open Access Initiative, Hungary advocating the open-access of scientific and scholarly knowledge. The Institute is a registered content provider under Open Access Initiative Protocol for Metadata Harvesting (OAI-PMH).

The journal is indexed \& included in WorldCat Discovery Service (USA), CrossRef Metadata Search (USA), WorldCat (USA), OCLC (USA), Open J-Gate (India), EZB (Germany) Scilit (Switzerland), Airiti (China), Bielefeld Academic Search Engine (BASE) of Bielefeld University, Germany, PKP Index of Simon Fraser University, Canada. 


\begin{abstract}
The paper aimed to examine students' perceptions of the features and tools in GeoGebra. A GeoGebra lesson was conducted involving 43 high school students. The questionnaires were employed to collect the research data. Descriptive statistics were used to gauge students' perceptions toward the software during a GeoGebra lesson. The findings showed that the students' perceptions toward GeoGebra features for the basic construction of geometry, the transformation angle, and the functions and exporting of images with a mean of 3.87, 3.69, and 4.15. Students' perceptions toward coordinates and equations were at a very easy level, with a mean of 4.34. The findings indicate that students have positive perceptions about the use of GeoGebra. Therefore, GeoGebra should be used as an alternative to promoting the use of technology in the teaching and learning of mathematics.
\end{abstract}

Keywords: GeoGebra, dynamic mathematics software, mathematics education, educational technology, teaching and learning of mathematics, information technology.

\title{
Introduction
}

In recent years, the urgent challenge facing the education sector is a thorough renovation of the content of the curriculum, teaching methods... to improve the quality of education and training. Regarding the innovation of teaching methods, the task is required as "Strongly renovating teaching and learning methods in a modernized direction, promoting student activity and eagerness in the classroom ... annihilation of superannuated means of imposing transmission methods based on a teacher-oriented approach, passive and non-creative memorization." (Vietnam Central Party Executive Committee, 2013). This task is also clearly stated in the new mathematics school program (2018) as "... organizing the teaching-oriented process, students are invited to explore, discover and make an inference to solve problems" (Ministry of Education and Training, 2018). At the same time, increasing the effective application of information technology (IT) in teaching is always highly focused. Instructions 29/2001/CTBGD\&ĐT emphasize "... using IT as the most effective supporting tool for innovating teaching and learning methods in all subjects" (Ministry of Education and Training, 2001). Similarly, in the high school mathematics education program in 2018 , the requirement is "... increasing the use of IT ... appropriately and effectively" (Ministry of Education and Training, 2018).

\section{Literature Review}

IT can become a useful tool to support teachers and students in teaching Maths. Researcher Nguyen Ba Kim and colleagues asserted: "Electronic computers can also be utilized in teaching to improve teaching methods, from which the quality of education can be enhanced as well" (Nguyen Ba Kim \& Vu Duong Thuy, 2004). Similarly, the author has also demonstrated that the application of in teaching process will change the teaching environment and have a strong impact on all elements of the teaching process Dao Thai Lai (2006), Nguyen Thi Nga (2016) emphasizes that IT tools allow:

- Enable abrupt representation of a problem or a concept to give it a meaning and create conditions for students to gain control over them.

- Connect different mathematical aspects (Algebra, Geometry, Spreadsheets ...) of the same concept or situation.

- Explore situations by making different shapes appear in a dynamic state.

- Generate conjectures from an interactive experiment when studying a problem containing open questions or a certain complexity and when making the first verification.

- Focus on solving life-related problems where calculations are often long and complicated.

- Conduct a quick assessment of some of the results received.

Thereby, the appropriate application of IT will bring positive effects to the teaching process, which contributes to the change with the teachers' teaching methods and makes students' learning more positive and proactive.

The application of IT in teaching is deployed at different levels depending on the level of awareness, IT skills of the teachers, the conditions of facilities, IT equipment of the schools. According to Dao Thai Lai (2006), the teachers of high schools mainly apply IT in teaching at the following levels: 
- Level 1: Apply IT to help teachers with some career tasks.

- Level 2: Apply IT to support one stage in the teaching process.

- Level 3: Apply IT to support the organization of teaching activities on a number of topics, according to the program or in typical Math teaching situations.

- Level 4: Integrate IT into the entire teaching process.

- Level 5: Change all traditional concepts, introduce a new school model in an IT-abundant environment: smart schools with e-learning training.

According to Le Van Tien (2009) the application of IT in teaching today usually only stops at two levels:

- Level 1: Replace the function of blackboard, white chalk;

- Level 2: Create specific and realistic visuals about mathematical objects or the operation process on objects being studied in the lesson. This facilitates students to acquire mathematical knowledge which is typically abstract.

Le Thai Bao ThienTrung (2011) divided the level of IT application in teaching Mathematics into three levels, including:

- Level 1: Teacher applies IT only for demonstration and demonstration.

- Level 2: Teacher applies IT to illustrate activities.

- Level 3: Students directly manipulate the software in a problem-suggesting situation.

Accordingly, the author thinks that "the application of IT at level 1 may favour the non-active teaching methods" (Le Thai Bao Thien Trung, 2011). If teachers apply IT only at level 1, IT only plays the role of modern means to support teachers in the teaching process, not for students. If teachers overuse IT, they sometimes reduce students' ability to abstract thinking math concepts. In addition, mathematics is an important subject that contributes to the development of student thinking skills, especially logical thinking, which often manifests in abundance when carried out by students themselves.

At level 2, teachers will prepare teaching activities on software. After that, teachers conduct teaching in class, manipulate software and ask questions for students. Students observe the results generated by the software when the teacher manipulates to answer questions. Here, teachers will use IT in combination with a particular teaching method to conduct the teaching process.

At level 3, the teacher is the person who organizes problem-suggesting situations and then delegates them to students. When the problems in situations become students' problems or tasks, they will perform manipulations in the software environment to find answers or make guesses. Note that the teacher only introduces how to use some functions of the software and students will self-coordinate the already-known functions into a tool to solve the assigned tasks voluntarily.

However, in the current math textbooks (textbooks), only pocket computers are mentioned explicitly and with instructions for use, with practice attached to some topics. On the other hand, the use of software and teaching programs depends on themselves and the experience of each teacher and student. The question to ask is: which teaching software to choose to effectively innovate teaching methods for teachers and learning outcomes of students?

GeoGebra - a dynamic math software, "an environment that combines dynamic geometry, algebra, calculus, and spreadsheet functions into one simple and easy-to-use package" (M. Hohenwarter\& Jones, 2007). Markus Hohenwater wrote GeoGebra in 2001 with the philosophy of open-source software. It is compatible with different operating systems. GeoGebra is widely used by the user community and developed from many countries around the world (Tatar, 2013a). Nguyen Phu Loc (2014), Le Tuan Anh (2010) show that GeoGebra is suitable and easy to use for Vietnamese teachers because GeoGebra is a free and simple software to be extensively applied. There is also proficiency in using GeoGebra in math teaching. 
For this study, the authors explore students' perceptions of how easy it is to use GeoGebra's functional tools after each instructional training session.

\section{Material and Methods}

In order to practically figure out how difficult or easy to use functions of GeoGebra software for teachers, a survey study was conducted through the course "Guide to using GeoGebra software".

This study uses a quantitative survey method using questionnaires to collect data. Descriptive statistics were used to evaluate students' perception of software in GeoGebra tutorial sessions. The survey tool is a revised questionnaire from Preiner (2008), ranging from very difficult to very easy levels of questions. The study was conducted with the help of 43 students from Pacific high schools at the beginning of the semester I, the school year 2015 - 2016.

Students were introduced and guided to learn the use and manipulation of functional tools as well as the potential of GeoGebra in learning Mathematics. At the end of the course, learners can: identify the functionality of each of the tools included in GeoGebra; construct "animated" figures; solve problems with GeoGebra support.

The structure and content of the sessions include:

Table 1: Structure and content of lessons

\begin{tabular}{ll}
\hline & \multicolumn{1}{c}{ Activity's contents } \\
\hline Lesson I: Basic geometric constructions & Activity 1: Line Bisector with paper \\
& Activity 2: Line Bisector with GeoGebra \\
& Activity 3: Square \\
Activity 4: Circumscribed Circle of a Triangle & Activity 5: Equilateral Triangle \\
Lesson II: The angle, transformations and pictures & Activity 1: Parallelogram with Angles \\
& Activity 2: Drawing Tool for Symmetric Figures \\
& Activity 3: Inserting a Background Image \\
Lesson III: Coordinates and equations & Activity 4: Rotation of a Polygon \\
& Activity 1: Coordinates of Points \\
& Activity 2: Linear Equations \\
Lesson IV: Functions and export of images & Activity 3: Slope Triangle \\
& Activity 4: Quadratic Equation \\
& Activity 1: Polynomials Functions \\
& Activity 2: Library of Functions \\
& Activity 3: Tangent and Slope Function \\
& Activity 4: Export of Static Pictures \\
\hline
\end{tabular}

\section{Results and Discussion}

Table 2 shows that Cronbach's Alpha scale reliability value is greater than 0.7 , which is within the range accepted by Nunally, 1978; Peterson, 1994; Slater, 1995 (cited by Hoang and Chu, 2006). The scaled average (ease of use) is divided into four sections. Difficult level is from 1.81 to 2.60 (difficult), the average level is 2.61 to 3.40 (medium), the easy level is 3.41 to 4.20 (easy) and very easy level is 4.21 to 5.0 (very easy ).

Table 2: Reliability coefficient of each part of the questionnaire

Part of the Questionnaire

BH.I.G: Characteristic of GeoGebra (Introduction)

BH.I : Basic geometry construction

BH.II: Angle, transformation and insert image

BH.III: Coordinate and equation

BH.IV: Function and export image
Cronbach alpha

0.918

0.924

0.927

0.873

0.943 
Table 3 shows the average value of the perceptions of students in the GeoGebra lessons. The results denote that the student's opinion about GeoGebra's features is at the ease of use $(\mathrm{M}=3.87)$. For the first lesson (The basic geometry construction tools) is easy to use $(\mathrm{M}=3.69)$. The results showed that student's concepts were that GeoGebra was easy to use in GeoGebra's feature and tools showcase. The second lesson (angle tools, transformations and image insertion) are also easy to use $(\mathrm{M}=4.00)$. For the third lesson (the coordinate system and the equation) is on average $(\mathrm{M}=4.34)$. The fourth session (functions and photo publishing) is also easy $(\mathrm{M}=4.15)$.

Table 3: Mean of teachers' perceptions toward GeoGebra workshop

\begin{tabular}{lccc}
\hline \multicolumn{1}{c}{ Part of the Questionnaire } & N & M & S.D \\
\hline BH.I.G: Characteristic of GeoGebra (Introduction) & 43 & 3.87 & 0.85 \\
BH.I : Basic geometry construction & 43 & 3.69 & 0.75 \\
BH.II: Angle, transformation and insert image & 43 & 4.00 & 0.69 \\
BH.III: Coordinate and equation & 43 & 4.34 & 0.70 \\
BH.IV: Function and export image & 43 & 4.15 & 0.84 \\
\hline
\end{tabular}

Table 4 shows the results of students' perception of GeoGebra's features in the introduction. Functional tools rated for very easy to use $(\mathrm{M}=4.21)$ are BH.I.G2 (Construction protocol) while other tools are evaluated by students as easy to use from 3.53 to 4.12 . Overall, Table 4 also shows that students rated GeoGebra's tools and features as easy to use.

Table 4: Students' awareness toward characteristics of GeoGebra in the Introductory Lesson

\begin{tabular}{llll}
\hline & & M & S.D \\
\hline BH.I.G1 & Construction protocol & 4.12 & .823 \\
BH.I.G2 & Navigation bar & 4.21 & .833 \\
BH.I.G3 & Rename objects & 3.70 & .914 \\
BH.I.G4 & Context menu & 3.95 & .925 \\
BH.I.G5 & Properties dialogue & 3.53 & .882 \\
BH.I.G6 & Grid & 3.70 & .860 \\
BH.I.G7 & Point capturing & 3.65 & .923 \\
BH.I.G8 & Trace of an object & 4.00 & .690 \\
BH.I.G9 & Background image & 4.00 & .951 \\
BH.I.G10 & Labeling objects & 3.93 & .768 \\
BH.I.G11 & Redefining objects & 3.88 & .762 \\
BH.I.G12 & Auxillary objects & 3.74 & .848 \\
BH.I.G13 & Insert static text & 4.00 & .787 \\
BH.I.G14 & Insert dynamic text & 3.91 & .921 \\
BH.I.G15 & Create a point on an object & 3.74 & .978 \\
Overall & 43 & 3.87 & \\
\hline
\end{tabular}

Table 5 shows students' perceptions of the features and tools guided in basic geometry construction lessons. The results show that all the functional tools are evaluated by students as easy to use with the lowest level being $\mathrm{M}=3.49$ and the highest level being $\mathrm{M}=3.95$. Overall, it can be inferred that students using GeoGebra's functional tools validate them to be easy.

Table 5: Students' awareness level in the first lesson

\begin{tabular}{llrc}
\hline & & M & S.D \\
\hline BH.I.1 & Line bisector construction with GeoGebra & 3.49 & .935 \\
BH.I.2 & Ortocenter of a triangle construction & 3.49 & .935 \\
BH.I.3 & Square of a segment & 3.70 & .860 \\
BH.I.4 & Segment through two points & 3.86 & .639 \\
BH.I.5 & Circle with center through points & 3.74 & .848 \\
BH.I.6 & Intersect two objects & 3.95 & .532
\end{tabular}




\begin{tabular}{llrr} 
BH.I.7 & Line through two points & 3.70 & .638 \\
BH.I.8 & Move & 3.70 & .773 \\
BH.I.9 & Polygon & 3.65 & .650 \\
BH.I.10 & Line bisector & 3.74 & .693 \\
BH.I.11 & Show/hide object & 3.65 & .842 \\
BH.I.12 & Move drawing pad & 3.65 & .720 \\
BH.I.13 & Zoom in... zoom out & 3.63 & .691 \\
BH.I.14 & Perpendicular line & 3.84 & .754 \\
Overall & 43 & & \\
\hline
\end{tabular}

Table 6 shows the perception of students about tools from BH.II.1 to BH.II.14 through activities in the second lesson. Tools tested by students that are very easy to use are primarily in items BH.II.1 (Intersect Two Objects) with an average scale of $\mathrm{M}=4.41$, items BH.II.10 (Polygon) and BH.II.16 (Rotate around point) with an average of coal calculated $\mathrm{M}=4.20$, items BH.II.15 and BH.II.17 with an average scale of $\mathrm{M}=4.25$. In which, item BH.II.1 Intersect two objects) is considered by students to be very easy to use and has the highest average scale of $M=4.41$. The students evaluated the tools in the remaining sections as easy to use with the lowest scale average of 3.41 in BH.II.8 (Line through two points) and the highest level of 4.16 in item BH.II.11 (Show hide objects).

Table 6: The level of students' awareness of the second lesson

\begin{tabular}{llrr}
\hline & & M & S.D \\
\hline BH.II.1 & Intersect two objects & 4.41 & .663 \\
BH.II.2 & Symmetry construction & 3.81 & .906 \\
BH.II.3 & Background image and axis of symmetry & 3.46 & .908 \\
BH.II.4 & Rotation of a polygon & 3.14 & .804 \\
BH.II.5 & Segment through two points & 3.41 & .851 \\
BH.II.6 & Circle with center through point & 3.90 & .946 \\
BH.II.7 & Parallelogram and angles & 3.55 & .881 \\
BH.II.8 & Line through two points & 4.11 & .730 \\
BH.II.9 & Move & 4.14 & .833 \\
BH.II.10 & Polygon & 4.20 & .741 \\
BH.II.11 & Show/hide object & 4.16 & .721 \\
BH.II.12 & Parallel line & 4.04 & .785 \\
BH.II.13 & Angle & 4.16 & .871 \\
BH.II.14 & Mirror at line & 4.11 & .762 \\
BH.II.15 & New point & 4.25 & .658 \\
BH.II.16 & Rotate around point & 4.20 & .674 \\
BH.II.17 & Insert image & 4.25 & .658 \\
Overall & 43 & & \\
\hline
\end{tabular}

Table 7 shows the perceptions of students about the ease of using GeoGeobra's functional tools in learning related to the hypothesis and the coordinate method. Items BH.III.2 (Slope intercept form of linear equations), BH.III.3 (Slope triangle), BH.III.6 (Parabola) are considered by HS to be easy to use with the average points scored on corresponding scales are 4.00, 4.07 and 4.11. Students evaluate the remaining items as very easy to use. In which, the item with the highest level of ease of use is BH.III.14 (Show/Hide Objects) with an average score of $\mathrm{M}=4.51$ and the item with the lowest level of ease of use is BH.III.12 (New point) with average $\mathrm{M}=4.25$.

Table 7: Students' perceptions of the third lesson

\begin{tabular}{llll}
\hline & & M & S.D \\
\hline BH.III.1 & Coordinates of points & 4.48 & .702 \\
BH.III.2 & Slope intercept form of a linear equation & 4.00 & .845 \\
BH.III.3 & Slope triangle & 4.07 & .668
\end{tabular}




\begin{tabular}{llll} 
BH.III.4 & Line through two points & 4.37 & .724 \\
BH.III.5 & Intersect two objects & 4.53 & .630 \\
BH.III.6 & Parabola & 4.11 & .762 \\
BH.III.7 & Move & 4.30 & .708 \\
BH.III.8 & Polygon & 4.48 & .702 \\
BH.III.9 & Slope triangle & 4.23 & .750 \\
BH.III.10 & Perpendicular line & 4.48 & .668 \\
BH.III.11 & Parallel line & 4.37 & .655 \\
BH.III.12 & New point & 4.25 & .658 \\
BH.III.13 & Slider & 4.44 & .547 \\
BH.III.14 & Show/hide object & 4.51 & .668 \\
BH.III.15 & Insert text & 4.46 & .591 \\
BH.III.16 & Slope & 4.48 & .798 \\
BH.III.17 & Vertex & 4.32 & .747 \\
Overall & 43 & & \\
\hline
\end{tabular}

Table 8 shows students' perceptions of the ease of use of GeoGebra's tools related to function topics, function graphs, and image publishing. The results showed that the tools in items from BH.IV.1 to BH.IV.13 were evaluated by students as easy to use. In addition to the sections BH.IV.4 (Image Publishing), BH.IV.5 (Inserting pictures into word) and BH.IV.8 Test) are easy to use, the rest are rated as very easy to use. In which, students evaluate item BH.IV.1 (Polynomial functions) as the easiest to use, with the corresponding average score of 4.44, followed by the item BH.IV.2 (Library of functions) and the item BH.IV.3 (Tangent of the functional graph).

Table 8: Students' perceptions of the fourth lesson

\begin{tabular}{llrc}
\hline & & M & S.D \\
\hline BH.IV.1 & Polynomial functions & 4.44 & .700 \\
BH.IV.2 & Library of functions & 4.37 & .900 \\
BH.IV.3 & Tangent to a function graph & 4.35 & .813 \\
BH.IV.4 & Export of pictures & 3.98 & .963 \\
BH.IV.5 & Inserting pictures into word & 3.98 & .938 \\
BH.IV.6 & Intersect two objects & 4.19 & .880 \\
BH.IV.7 & Move & 4.16 & .721 \\
BH.IV.8 & Root & 3.93 & .936 \\
BH.IV.9 & Perpendicular line & 4.14 & .833 \\
BH.IV.10 & New point & 4.09 & .895 \\
BH.IV.11 & Tangent & 4.05 & .844 \\
BH.IV.12 & Show/hide object & 4.23 & .751 \\
BH.IV.13 & Extreme & 4.09 & .750 \\
Overall & 43 & & \\
\hline
\end{tabular}

An overview of students' conception for the use of GeoGebra's feature tools is that this tool is relatively easy to be used, regarding the context of high schools. In activities in the first session, students initially familiarize themselves with the activities in GeoGebra classes and use the GeoGebra tool easily, although the average point of the scale is 3.87 for the introduction class of tools (Introduction characteristic of GeoGebra) and 3.69 for the guidance-to-use class of tools (Basic geometry construction). Since the second session, students have become familiar with the interface and effective manipulation of functional tools with the scale point above 4.00 .

\section{Conclusion}

The survey results show that students can easily access and use GeoGebra software. From those results mentioned above, it can be said that the deployment of using GeoGebra software to support maths teaching and learning is feasible and enforceable, especially to help students to self-study and self-explore the relationships among objects in math fields. Thanks to GeoGebra, teachers can diversify teaching methods 
to create opportunities for students to understand the knowledge of Mathematics through activities of experience and exploration. As open-source software, GeoGebra can be widely used in the community of learners and teachers, especially in developing regions such as the provinces in the Mekong Delta, Vietnam.

\section{References}

[1]. Vietnam Central Party Executive Committee, 2013. Resolution No. 29-NQ/TW on radical and comprehensive renovation of education and training to meet requirements of modernization and industrialization in the socialist-oriented market economy and international integration. Available from https://moet.gov.vn/tintuc/Pages/doi-moi-can-ban-toan-diengd-va-dt.aspx?ItemID=3928

[2]. Ministry of Education and Training, 2001. Instructions No. 29/2001/CT-BGD\& ĐT on strengthening teaching, training and application of information technology in the education sector in the period 2001-2005. Available from http://vbpl.vn/bogiaoducdaotao/pages/vbpq-luocdo.aspx?ItemID=79582

[3]. Ministry of Education and Training, 2018. Mathematics education program for secondary education. Available from https://data.moet.gov.vn/index.php/s/m6ztfi7sUIIGQdY\#pdfviewer

[4]. Le Tuan Anh, 2010. Developing Vietnamese pre-service high school mathematics teachers' skills of using Geogebra. GeoGebra International Journal of Romania. Vol.4 No.1

[5]. Hoang Trong and Chu Nguyen Mong Ngoc, 2006. Analysis of research data with SPSS. Ho Chi Minh City University of Economics. Hong Duc Publishing House.

[6]. Dao Thai Lai, 2006. Application of information technology in teaching in Vietnamese schools. The VietNam National Institute of Education Sciences. Ha Noi.

[7]. Nguyen Thi Nga, 2016. Potential and Advantatages of dynamic geometry software Cabri in teaching and learning Mathematics: the case of teaching the notion of function. Journal of Science of HNUE. Educational Sci., 2016, Vol.61, No. 6, pp. 60-6.8. DOI: 10.18173/2354-1075.2016-0049

[8]. Hohenwarter, M., \& Jones, K, 2007. Ways of linking geometry and algebra: The case of GeoGebra. Proceedings of the British Society for Research into Learning Mathematics, 27, 126-131.

[9]. Loc, N. P., \&Triet, L. V. M., 2014. Dynamic software "GeoGebra" for teaching mathematics: Experiences from a training course in Can Tho University. European Academic Research. ISSN: 2286 - 4822. Vol. II, Issue 6 (2014), pp.7908-7920.

[10]. Le Van Tien, 2009. Applying Information Technology contributes to the innovation in teaching and learning mathematics at high school education. Journal of Science. Ho Chi Minh University of Education. No. 50, 2009, p. 125-134.

[11]. Le Thai Bao Thien Trung (2011). Issues of application of information technology to teaching mathematics and the benefits of calculators. Journal of science. Ho Chi Minh University of education. No. 30, 2011, p. 51-58.

[12]. Tatar, E., 2013a. The Effect of Dynamic Software on Prospective Mathematics Teachers' Perceptions Regarding Information and Communication Technology. 38(12).

[13]. Preiner, J., 2008. Introducing dynamic mathematics software to mathematics teachers: The case of GeoGebra. $\mathrm{PhD}$ Thesis, University of Salzburg Austria.

[14]. Nguyen Ba Kim \& Vu Duong Thuy, 2004. Method of teaching Mathematics. University of Education Publishing House. Ha Noi. 